\title{
Sarcomatoid carcinoma of the stomach: A case report and literature review
}

\author{
CHUN-CHAO ZHU, MAO-RAN LI, TIAN-LONG LIN and GANG ZHAO \\ Department of Gastrointestinal Surgery, Ren Ji Hospital, School of Medicine, \\ Shanghai Jiao Tong University, Shanghai 200127, P.R. China
}

Received September 23, 2014; Accepted April 30, 2015

DOI: $10.3892 / \mathrm{ol} .2015 .3460$

\begin{abstract}
Sarcomatoid carcinoma of the stomach is a rare type of malignant tumor, characterized by distinct cellular morphology. This type of tumor is even more rare in giant size. The present study reports a case of giant sarcomatoid carcinoma, which developed in the distal stomach. A 49-year-old male underwent medical investigation for gastrointestinal hemorrhage. Endoscopic examination, computed tomography (CT) and positron emission tomography-CT scan identified a giant neoplasm, which involved the gastric antrum and body, gallbladder and hepatic flexure of the colon. Surgery was performed to excise the tumor, which was $\sim 14 \times 13 \times 8 \mathrm{~cm}$ in size. A diagnosis of sarcomatoid carcinoma was made since the tumor was positive for epithelial markers, even within the mesenchymal elements. To the best of our knowledge, only 5 cases of sarcomatoid carcinoma of the stomach have been previously reported, and a tumor that has been able to be resected despite such a large size has never been reported.
\end{abstract}

\section{Introduction}

Sarcomatoid carcinomas are a rare type of tumor, particularly in the stomach (1). Snover et al (2) first described the features of sarcomatoid carcinoma. A diagnosis of sarcomatoid carcinoma should be made when the sarcomatous component of the tumor mainly consists of spinal cells, but the cells are morphologically or immunologically identifiable as epithelial cells (3). Occasionally the tumors exhibit specific, notable differentiation, for example osteoclastic-like giant cells (4). Due to the poor efficacy of chemotherapy and radiotherapy, surgery remains the most effective treatment $(5,6)$. The present report presents details of a large-size sarcomatoid carcinoma in the stomach, which was completely resected by surgery. In addition, complete clinical data is included.

Correspondence to: Dr Gang Zhao, Department of Gastrointestinal Surgery, Ren Ji Hospital, School of Medicine, Shanghai Jiao Tong University, 1630 Dongfang Road, Shanghai 200127, P.R. China E-mail: zhaogga@126.com

Key words: immunohistochemistry, sarcomatoid carcinoma, stomach

\section{Case report}

A 49-year-old Chinese male with melena, anemia and fatigue visited the Emergency Room at Ren Ji Hospital Affiliated to Shanghai Jiao Tong University School of Medicine (Shanghai, China), on July 22, 2013. The proband's hemoglobin was $39 \mathrm{~g} / 1$ upon entering the ward. Abdominal ultrasonography revealed a mass with mixed echogenicity in the right upper quadrant of the abdomen. A computed tomography (CT) scan revealed a $14 \mathrm{~cm}$-diameter mass in the gastric antrum and gastric body, which infiltrated the serosa of the stomach. The gallbladder and hepatic flexure of the colon were also involved. CT contrast enhancement was evident in the two lesions (Figs. 1 and 2). A positron emission tomography (PET)-CT scan revealed a large irregular mass with abnormally high fluorodeoxyglucose (FDG) metabolism in the region of the distal stomach, gallbladder fossa and hepatic tissue in the vicinity (Fig. 3). A lymph node with high FDG metabolism was observed in the region of the pancreatic head (Fig. 4). Endoscopic investigation of the upper gastrointestinal tract identified an irregularly shaped lesion on the gastric antrum and body (Fig. 5). The histopathological section of the endoscopic biopsy revealed that the majority of the tumor cells were spindle shaped in morphology. Following a primary diagnosis of gastric malignancy, the patient received surgery on August 3,2013. A 14x13x8 cm, solid mass with an irregular surface was identified during surgery. In addition, the No. 13 lymph nodes were markedly enlarged and fused, measuring 20x35 mm. The distal stomach, gallbladder and right hemicolon were resected in order to completely remove the tumor. In addition, an extended D2 lymphadenectomy was also performed. Reconstruction was conducted with the gastrojejunum Roux-en-Y anastomosis (7). Histopathological diagnosis was as follows: Sarcomatoid carcinoma of the gastric antrum and body $(14 \times 13 \times 8 \mathrm{~cm}$ in size, with serosa invasion), the gallbladder and adjacent colon wall were also involved and No. 13 lymph node metastasis was observed.

Following surgery, the patient recovered successfully and was discharged from hospital on August 19, 2013. However, the tumor recurred and the patient succumbed to the disease in February 2015.

Pathological findings. Macropathological analysis of the resected specimen revealed a mass with an irregular surface, 


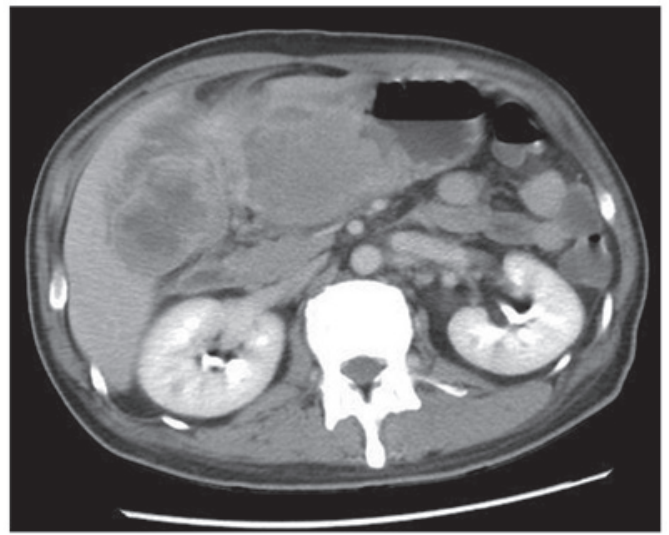

Figure 1. Computed tomography scan data revealed a $14 \mathrm{~cm}$-diameter mass in the gastric antrum and body, which infiltrated the serosa of the stomach In addition, the gallbladder and hepatic flexure of the colon were involved.

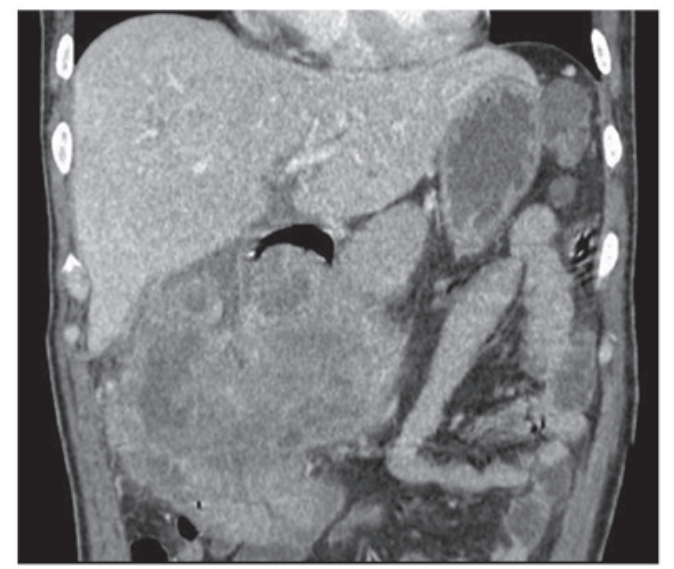

Figure 2. A coronal computed tomography scan of the tumor.

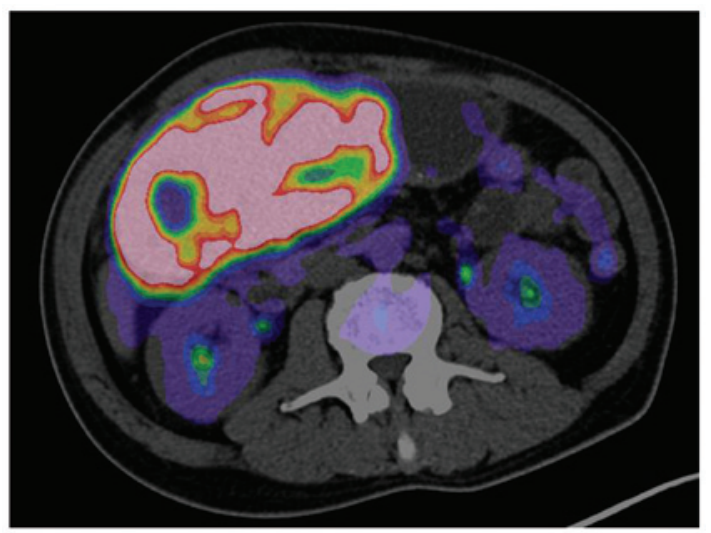

Figure 3. Positron emission tomography-computed tomography scan revealed a large irregular mass with abnormally high fluorodeoxyglucose metabolism in the region of distal stomach, gallbladder fossa and hepatic tissue in the vicinity.

measuring 14x13x8 cm (Fig. 6). The cross-sectional surface of the tumor was gray-white in appearance. Paraffin-embedded, 4-6- $\mu$ m-thick specimens were mounted on microscopic slides. Following deparaffinization, tissue samples were postfixed in buffered osmium tetroxide, dehydrated in ethanol and embedded. Ultrathin sections were prepared and examined

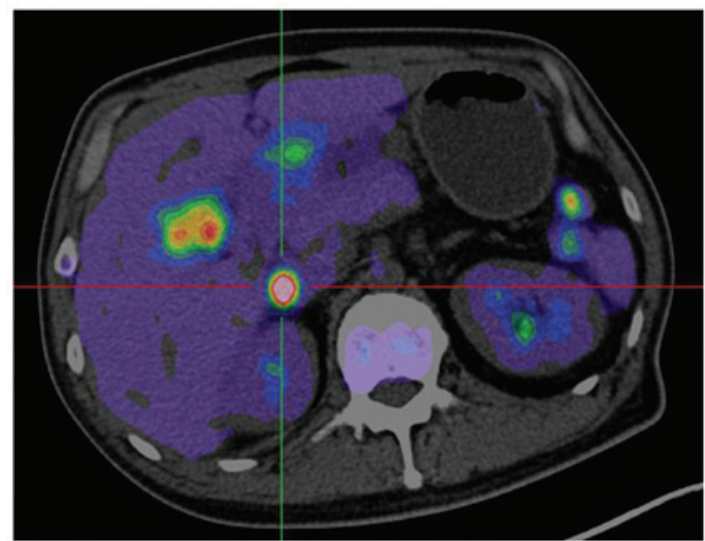

Figure 4. A lymph node with high fluorodeoxyglucose metabolism was observed in the region of the pancreatic head.

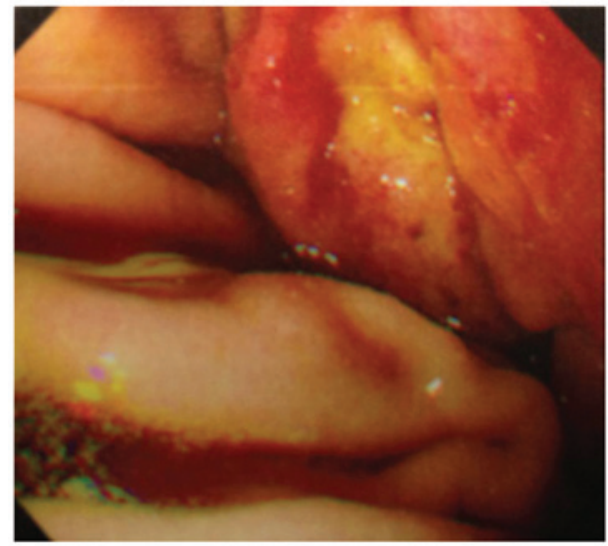

Figure 5. Endoscopic survey of the upper gastrointestinal tract identified an irregularly shaped lesion on the antrum and body.

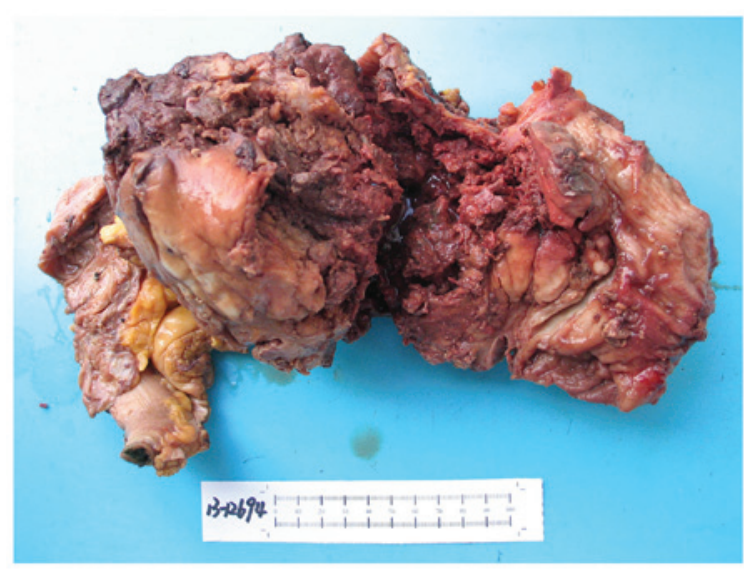

Figure 6. Macropathological findings of the resected specimen revealed a mass with an irregular surface, measuring $14 \times 13 \times 8 \mathrm{~cm}$ in size.

using a transmission electron microscope (Axio Imager A1; Carl Zeiss, Ltd., Cambridge, UK).

Micropathologically, the tumor cells of the stomach demonstrated infiltrative growth. The cytological characteristics of the tumor cells revealed overtly malignant features. Microscopic observation identified mainly spindle-shaped 
Table I. Primary antibodies and their marked cells, dilution, source, system and results in tumor cells.

\begin{tabular}{|c|c|c|c|c|c|}
\hline Antibody & Marker & Clonality & Source & Dilution & Result \\
\hline P63 & Tumor cell & Monoclonal & Dako & $1: 50$ & - \\
\hline $\mathrm{S} 100$ & Dendritic cell & Monoclonal & Dako & $1: 200$ & - \\
\hline SMA & Smooth muscle actin & Monoclonal & Dako & $1: 100$ & - \\
\hline Desmin & Muscle cell & Monoclonal & Dako & $1: 50$ & - \\
\hline CD68 & Pan-macrophage & Monoclonal & Dako & $1: 50$ & + \\
\hline Vimentin & Stroma cell & Monoclonal & Dako & $1: 100$ & + \\
\hline CD117 & Stroma cell & Monoclonal & Dako & $1: 50$ & - \\
\hline DOG-1 & Stroma cell & Monoclonal & Dako & $1: 50$ & - \\
\hline $\mathrm{Ki}-67$ & Antigen proliferation marker & Polyclonal & Dako & $1: 50$ & $80 \%$ \\
\hline CD34 & Vascular endothelial cell & Monoclonal & Dako & $1: 50$ & - \\
\hline CD31 & Vascular endothelial cell & Monoclonal & Dako & $1: 50$ & - \\
\hline Melan-A & Melanoma & Monoclonal & Dako & $1: 100$ & - \\
\hline HMB45 & Melanoma & Monoclonal & Dako & $1: 100$ & - \\
\hline CK & Epithelial cell & Monoclonal & Dako & $1: 50$ & + \\
\hline CK7 & Epithelial cell & Monoclonal & Dako & $1: 50$ & + \\
\hline CD20 & Epithelial cell & Monoclonal & Dako & $1: 20$ & - \\
\hline
\end{tabular}

All antibodies were raised in mice against human antigens. Dako, Glostrup, Denmark. DOG-1, discovered on GIST-1; HMB45, human melanoma black 45; CK, cytokeratin.

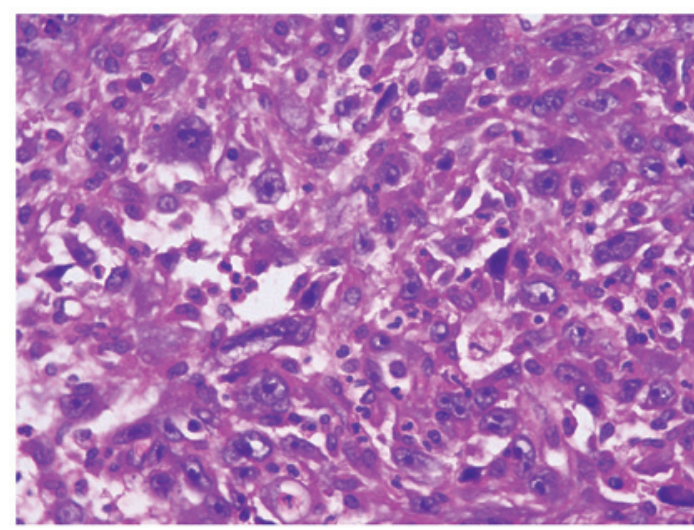

Figure 7. Tumor cells demonstrated spindle-shaped structures, significant atypical nuclei, pleomorphic nuclei and giant nuclei. Cells were stained with hematoxylin and eosin stain (magnification, $x 400$ ).

cell structures with significantly atypical, pleomorphic and giant nuclei. In addition, a minority area of adenocarcinoma, with papillary and tubular growth of cancer cells, was also detected. The sarcomatous and carcinomatous elements were frequently closely admixed with each other throughout the tumor. There were $>50$ mitoses per 10 high-power fields (Fig. 7).

Immunohistochemical (IHC) examination of the sections was performed using a standard avidin-biotin-peroxidase complex method (8). The antibodies used and the results obtained are summarized in Table I. The tumor cells were positive for Vimentin (Fig. 8), cytokeratin (CK), CK7 (Fig. 9) and CD68 (Fig. 10). Expression of the proliferation marker Ki-67 reached $80 \%$ (Fig. 11). The cells were negative for Desmin, CD20, CD31, CD34, CD117, discovered on GIST-1 (DOG-1),

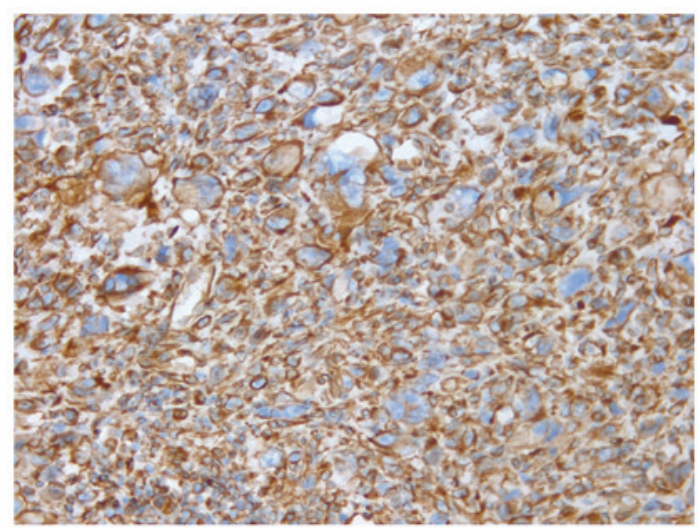

Figure 8. Tumor cells positive for Vimentin (magnification, x200).

S-100, ALDOG-1, P63, Melan-A and human melanoma black 45 (Table I).

Diagnosis of sarcomatoid carcinoma of the stomach was confirmed by these histomorphological and IHC findings.

\section{Discussion}

Adenocarcinoma, adenosquamous carcinoma and squamous carcinoma are more common types of tumor in stomach (9). Sarcomatoid carcinoma is a rare type of primary gastric tumor, and it is difficult to make a definitive pre-operative diagnosis in such cases. Reviewing the clinical data of the present case, further characters of gastric sarcomatoid carcinoma have been identified. The histopathological section of endoscopic biopsy revealed that the majority of the tumor cells were spindle shaped in morphology. In addition, minor elements with adenocarcinoma were also observed. In 


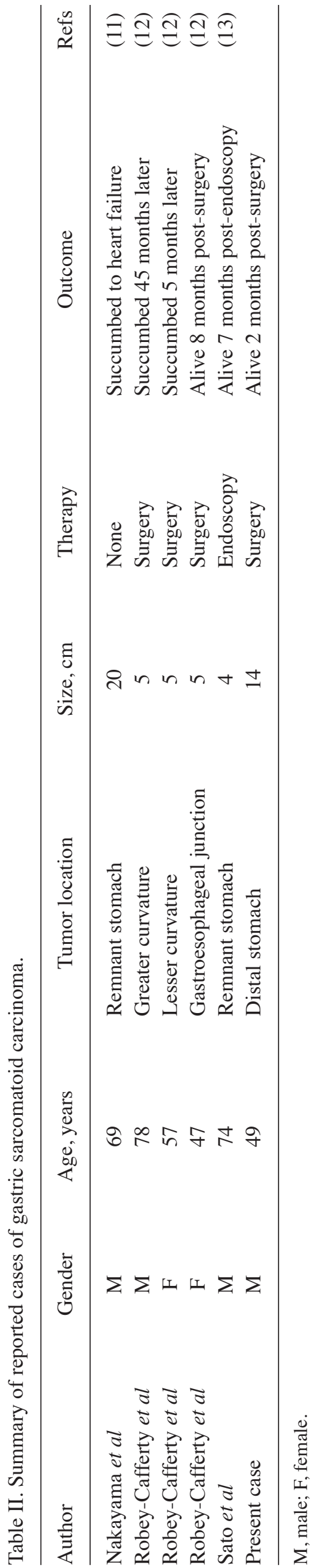

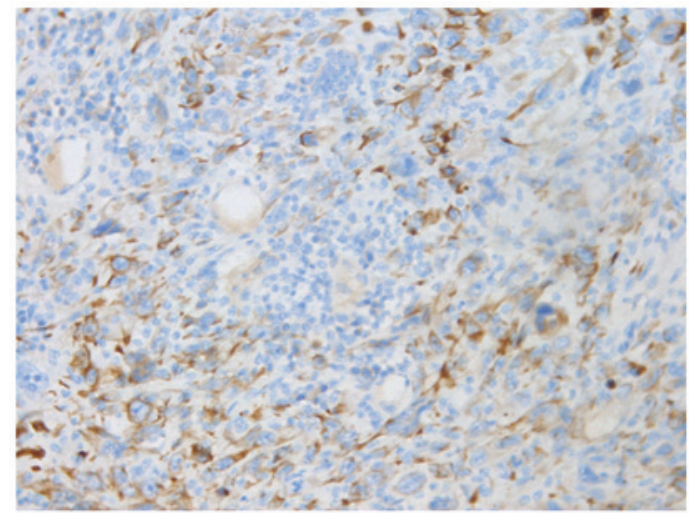

Figure 9. Tumor cells positive for cytokeratin 7 (magnification, x200).

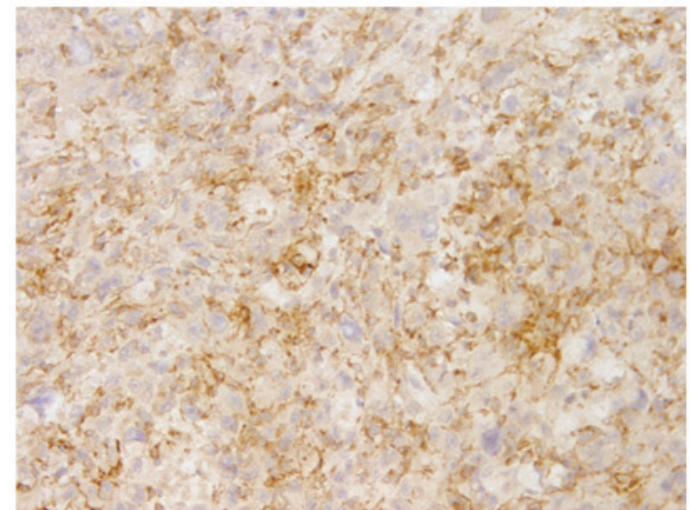

Figure 10. Tumor cells positive for CD68 (magnification, x200).

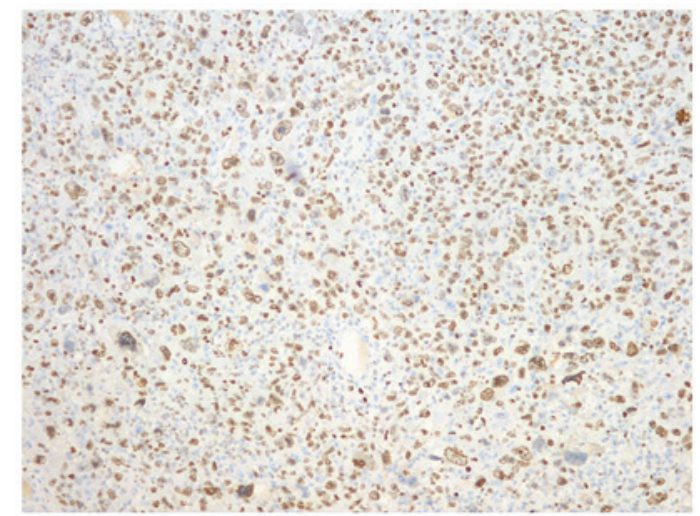

Figure 11. Tumor cells positive for Ki-67 (magnification, x200).

terms of radiography, PET-CT scans provided greater clarity in concluding correct diagnosis.

However, final diagnosis remains dependent on the results of the IHC exam $(6,10)$. The present tumor was finally diagnosed as sarcomatoid carcinoma, since IHC analysis was positive for epithelial markers in the mesenchymal elements of the tumor. Reviewing the current case and 5 previously reported cases from the literature (11-13), the characteristics of all reported cases are summarized in Table II. The age of the patients ranged from 47-78 years old (mean, 62.7 years). All previously reported tumors were located close to the gastroesophageal junction, whereas the present tumor was 
located in the distal stomach. A total of 5 out of the 6 patients from the literature, including the case reported in the present study, underwent surgical resection. The size of the tumor in the present case was the largest. In the sixth case, the patient with a $20-\mathrm{cm}$ diameter tumor died prior to surgical intervention due to respiratory failure. A total of 4 out of the 5 resectable cases, except for the present case, developed recurrence within several months, indicating that the prognosis of this disease is relatively poor.

The understanding of sarcomatoid carcinoma has been hindered by variations in nomenclature and classification. Multiple nomenclatures have been used in the previous literature, including carcinosarcoma, pseudosarcoma, pseudocarcinoma and spindle cell carcinoma. The multiple names demonstrate the varied understandings of this disease, which have resulted in complex definitions regarding this type of tumor.

It is highlighted in the current literature that sarcomatoid carcinomas display malignant epithelial and stromal components, and sarcoma components which occupy $>50 \%$ of the elements involved. Sarcomatoid cancer is able to develop in numerous locations, including the nasopharynx, lung, digestive tract, urinary and genital system $(3,5,10,14)$. Sarcomatoid cancer, in the skin or mucous membrane, presents similarly to polypus under a microscope, and IHC may be used to detect the presence of various types of sarcomatoid properties, for example clusters of well-differentiated cells, and demonstration of intraepithelial neoplasia without the element of cancer. The sarcomatoid cancer has elements of both cancer and sarcoma. In the immune phenotype analysis, the properties of the cancer part remain constant in CK and epithelial membrane antigen (EMA). By contrast, the phenotypes of sarcoma part vary. The positive rates of CK and EMA are $26 \%$ and $17.9 \%$, respectively (10).

The pathogenic mechanisms underlying sarcomatoid carcinoma remain to be elucidated. P53 mutations have been observed in this kind of tumor. Certain scientists have hypothesized that sarcomatoid carcinoma may be differentiated from stem cells (15), as the carcinomatous and sarcomatoid elements are monoclonal. Two of the previous cases of sarcomatoid carcinoma were discovered on remnant stomach. Studies have reported that a large proportion of sarcomatoid carcinomas developed in various organs following treatment for other diseases (16). For example, these tumors were found in the urinary system following transurethal resection or chemotheraphy, and in the liver following arterial embolization or radiofrequency ablation.

The prognosis of sarcomatoid carcinoma is poor, therefore the patient in the present case may present with an unfavorable prognosis. Furthermore, the large size of the tumor and the involvement of No. 13 lymph node metastases and adjacent organs decrease the probability of a favorable prognosis. The thoroughness of surgical resection is the most significant factor underlying patient survival. Although sarcomatoid carcinoma is insensitive to chemotherapeutic drugs (5), certain patients were demonstrated to benefit from chemotherapy with a carbopaltin and gemcitabine regime (17). However, whether or not to treat gastric sarcomatoid carcinoma with chemotherapy remains a controversial issue.

Reports on giant sarcomatoid carcinoma are infrequent. The present case of sarcomatoid carcinoma of the stomach therefore presented a rare opportunity for study. Resection of such a large size tumor presented difficulties, and it was a challenge to completely resect. The curative effect of adjuvant therapies for gastric sarcomatoid carcinoma remains unclear, and therefore follow-up of this patient is important for enhancing understanding of the disease.

\section{Acknowledgements}

The present study was supported by the Project of Science and Technology Commission of Shanghai Municipality (no. 13ZR1425200) and the Project of Shanghai Municipal Health Bureau (no. 20114241).

\section{References}

1. Carboni F, Levi Sandri GB, Valle M, Covello R and Garofalo A: Gastric sarcomatoid carcinoma. J Gastrointest Surg 17: 2025-2027, 2013.

2. Snover DC, Levine GD and Rosai J: Thymic carcinoma. Five distinctive histological variants. Am J Surg Pathol 6: 451-470, 1982.

3. Cheng L, Huang WB and Rao Q: Molecular pathology, histopathologic features and differential diagnosis of sarcomatoid carcinoma of urinary bladder. Zhonghua Bing Li Xue Za Zhi 42: 416-419, 2013 (In Chinese).

4. Yozu M, Glengarry J and Ahmed SS: Cutaneous squamous cell carcinoma associated with proliferation of osteoclast-like giant cells. J Pak Med Assoc 61: 922-925, 2011.

5. Huang SY, Shen SJ and Li XY: Pulmonary sarcomatoid carcinoma: A clinicopathologic study and prognostic analysis of 51 cases. World J Surg Oncol 11: 252, 2013.

6. Chien T, Chou J, Chang T and Lin C: Successful treatment of biphasic metaplastic sarcomatoid carcinoma of the breast by evaluation of immunohistochemical markers. Hematol Oncol Stem Cell Ther 3: 89-93, 2010.

7. Langhans P, Schönleben K and Bünte H: The routine use of Roux-en-Y anastomosis in gastric surgery. Scand J Gastroenterol Suppl 67: 247-249, 1981.

8. Charpin C,Martin PM,Lissitzky JC,Lavaut MN,KoppF, PourreauSchneider N, Jacquemier J, and Toga M: Immunocytochemical antigens detection in human breast carcinomas: A light and electron microscopy study using avidin biotin peroxidase and preembedding method. Cancer Detect Prev 8: 77-85, 1985.

9. Corso G, Seruca R and Roviello F: Gastric cancer carcinogenesis and tumor progression. Ann Ital Chir 83: 172-176, 2012.

10. Viswanathan S, Rahman K, Pallavi S, Sachin J, Patil A, Chaturvedi P, D'Cruz A, Agarwal J and Kane SV: Sarcomatoid (spindle cell) carcinoma of the head and neck mucosal region: A clinicopathologic review of 103 cases from a tertiary referral cancer centre. Head Neck Pathol 4: 265-275, 2010.

11. Nakayama Y, Murayaama H, Iwasaki H, Iwanaga S, Kikuchi M, Ikeda S, Okada M, Iizuka Y and Iwashita A: Gastric carcinosarcoma (sarcomatoid carcinoma) with rhacdomyoblastic and osteoblastic differentation. Pathol Int 47: 557-653, 1997.

12. Robey-Cafferty SS, Grignon DJ, RoJY, Cleary KR, Ayala AG, Ordonez NG, and Mackay B: Sarcomatoid carcinoma of the stomach. A report of three cases with immunohistochemical and ultrastructural observations. Cancer 65: 1601-1606, 1990.

13. Sato A, Oki E, Kohso H, Endo Y, Uchida H, Hiroshige S, Ishida M, Saito G, Matsumoto T, Takeuchi H, Kusumoto T, Yoshikawa Y and Muto Y: Sarcomatoid carcinoma of the remnant stomach: report of a case. Surg Today 43: 308-312, 2013.

14. Ren CL, Jin P, Han CX, Xiao Q, Wang DR, Shi L, Wang DX and Chen H: Unusual early-stage pancreatic sarcomatoid carcinoma. World J Gastroenterol 19: 7820-7824, 2013.

15. Znati K, Tadlaoui N, Bernoussi Z, Mikou A, Mahassini N, Jahid A, Mansouri F and Hachimi A: Biphasic sarcomatoid carcinoma of the thyroid: An exceptional localization of a rare tumor. Ann Endocrinol (Paris) 67: 64-68, 2006 (In French).

16. Badhiwala N, Chan R, Zhou HJ, Shen S and Coburn M: Sarcomatoid carcinoma of male urethra with bone and lung metastases presenting as urethral stricture. Case Rep Urol 2013: 931893, 2013.

17. Sugano T, Mori M, Namba Y, Uenami T, Kagami S and Yokota S: A case of sarcomatoid carcinoma of the lung successfully treated with carboplatin, paclitaxel and bevacizumab. Nihon Kokyuki Gakkai Zasshi 49: 304-308, 2011 (In Japanese). 